\title{
Does HPA-axis activity mediate the relationship between obstetric complications and externalizing behavior problems? The TRAILS study
}

\author{
Rianne Marsman · Judith G. M. Rosmalen • \\ Albertine J. Oldehinkel · Johan Ormel · \\ Jan K. Buitelaar
}

Received: 17 November 2008 / Accepted: 17 March 2009/Published online: 8 April 2009

(C) The Author(s) 2009. This article is published with open access at Springerlink.com

\begin{abstract}
To examine whether HPA-axis activity mediates the relationship between obstetric complications (OCs) and externalizing behavior problems, and to investigate whether this model is different for boys and girls. In a population-based cohort of 1,768 10- to 12-year-old early adolescents, we assessed the cortisol awakening response and evening cortisol levels. Externalizing behavior problems were assessed using the Child Behavior Checklist and the Youth Self-Report. OCs were retrospectively assessed in a parent interview. OCs significantly predicted externalizing behavior problems, but OCs did not predict HPA-axis activity. Thus, the mediation model was not supported. In addition to the relationship between HPA-axis activity and externalizing behavior problems, which is specific for girls, there is also a relationship between OCs and externalizing behavior problems. However, these two mechanisms are not related to each other indicating that HPA-axis activity is not
\end{abstract}

R. Marsman ( $₫)$. J. K. Buitelaar

Department of Psychiatry, Radboud University Nijmegen

Medical Centre, PO Box 9101, 6500 HB Nijmegen,

The Netherlands

e-mail: R.Marsman@psy.umcn.nl

J. G. M. Rosmalen · A. J. Oldehinkel · J. Ormel Interdisciplinary Center for Psychiatric Epidemiology, University Medical Center Groningen, University of Groningen, Groningen, The Netherlands

\section{A. J. Oldehinkel}

Department of Child and Adolescent Psychiatry,

Erasmus University Medical Center,

Sophia Children's Hospital Rotterdam,

Rotterdam, The Netherlands

J. K. Buitelaar

Karakter Child and Adolescent Psychiatry University Centre

Nijmegen, Nijmegen, The Netherlands a mediator in the relationship between OCs and externalizing behavior problems. Future research should focus on understanding the mechanism through which OCs cause externalizing behavior problems.

Keywords Early adolescence .

Externalizing behavior problems $\cdot$ Cortisol .

Pre- and perinatal risk factors - General population

\section{Introduction}

Externalizing behavior problems refer to a class of hyperactive and impulsive, oppositional defiant and aggressive behaviors that are very prevalent in children and adolescents and are associated with a huge burden for family and society [29]. Externalizing behavior problems have a multifactorial etiology, with a moderate to strong contribution of genetic risk factors [15], and additional contribution of environmental risk factors, such as family and parenting factors [26], and obstetric complications (OCs) $[5,7,10,21,35,40]$.

Several studies have revealed that OCs, such as maternal physical problems during pregnancy and delivery, prematurity, macrosomia (i.e. birth weight higher than 4,500 g), and acute anoxia or hypoxia, are involved in the etiology of externalizing behavior problems $[5,7,10,35,40]$. In addition, emerging evidence suggests that the psychological well being of the mother during pregnancy, such as maternal emotional difficulties during pregnancy, and prenatal stress or anxiety of the mother, are related to externalizing behavior problems $[5,21]$. Several composite scores on OCs have been introduced $[8,33,38]$ in which the classical biological risk factors have been combined with factors concerning 
the psychological well being of the mother during pregnancy. This composite score on OCs have also been related to externalizing behavior problems in previous studies, including a TRAILS study [7, 10]. However, despite the extensive literature on the relationship between OCs and externalizing behavior problems, little attention has been paid to the mechanism by which OCs may cause externalizing behavior problems. Several researchers suggested a mediating role of neurobiological deficits in the relationship between early childhood adversity and externalizing behavior problems [4, 19, 47]. In line with these researchers, Allen et al. [5] proposed a biological model in which neurobiological deficits may explain the relationship between OCs and externalizing behavior problems. Besides causing prefrontal damage, the effects of OCs may impact multiple other brain sites [40]. Among other neurobiological deficits, impairment of the hypothalamus-pituitary-adrenal (HPA)-axis has often been suggested as a potential mediator in the relationship between early stress (from before birth to early childhood) and externalizing behavior problems $[4,19,25,47]$.

Indeed, specific OCs, such as instrumental delivery (i.e. forceps or ventouse), meconium staining of the liquor, prematurity, and maternal stress or anxiety during pregnancy, may adversely affect the function of the child's HPA-axis [11, 24, 32, 36, 44]. The HPA-axis is known as a central component of the neuroendocrine stress system, and produces cortisol as its major end product [45]. On the one hand, several studies suggest that specific OCs are directly associated with elevated cortisol levels in the fetus, child or early adolescent $[11,24,32,36]$. On the other hand, several studies suggest that specific OCs are associated with elevated cortisol levels in response to a stressor in both infants and young adults [16, 44]. Moreover, maternal anxiety during pregnancy is associated with an elevated cortisol awakening response (CAR), that is, the rise in cortisol from awakening to $30 \mathrm{~min}$ later [36].

For mediation to occur, HPA-axis activity must also be associated with externalizing behavior problems. In previous TRAILS studies, it was demonstrated that elevated basal cortisol levels are associated with externalizing behavior problems in girls from the general population [30, 43]. Although seemingly contradictory, these findings may be congruent with the general idea that externalizing behavior problems are associated with lower basal cortisol levels $[4,47]$. That is, a large meta-analysis suggests that the effect size for this inverse association is small and specific for boys and for clinical samples [4]. Thus, this positive association may be specific for girls from the general population. Yet the study by van Bokhoven et al. [46] indicated that the positive association may also be inherent to the general population per se, revealing a positive association for boys from the general population. In addition, Pajer et al. [37] found an inverse association between HPA-axis activity and externalizing behavior problems in a clinical sample of girls, also indicating that the potential positive association is specific for girls with externalizing behavior problems from the general population.

Two main bodies of evidence suggest that the mediation model is gender-specific. First, the relationship between HPA-axis activity and externalizing behavior problems is different for boys and girls. As said before, there is evidence that the inverse association between HPA-axis activity and externalizing behavior problems is specific for boys [4], whereas the positive association between HPAaxis activity and externalizing behavior problems is specific for girls [30, 43]. In addition, there may be gender differences in the level of HPA-axis activity. More specifically, gender may be associated with HPA-axis activity, in that girls may show higher cortisol levels than boys $[27,41]$. Furthermore, there are gender differences in the level of externalizing behavior problems. Boys exceed girls in rates of a life course persistent pattern of externalizing behavior problems, while gender differences are small in an adolescent-limited pattern of externalizing behavior problems [34]. Second, there may be gender differences in the sensitivity to OCs. Whereas boys experience more OCs than girls [42] and are more vulnerable to the effects of OCs than girls [7, 28], the relationship between OCs and externalizing behavior problems is possibly specific for girls [5]. However, there is also evidence that there are no gender differences in the relationship between OCs and externalizing behavior problems [7]. In addition, the TRAILS study that found a relationship between OCs and externalizing behavior problems in our sample did not investigate gender differences [10]. In conclusion, gender specificity of the relationship between OCs and externalizing behavior problems is still open to question.

In summary, there are empirical and theoretical reasons to hypothesize that HPA-axis activity serves as a mediator in the relationship between OCs and externalizing behavior problems. Our hypotheses are partly based on the previous TRAILS studies that revealed that OCs predict externalizing behavior problems, and that HPA-axis activity is associated with externalizing behavior problems in girls $[30,43]$. Therefore, we hypothesize that HPA-axis activity may be the linking mechanism between OCs and externalizing behavior problems. However, to date, no study investigated this model. The mediation model predicts that OCs lead to higher cortisol levels, which in turn lead to more externalizing behavior problems. We hypothesize that this model is specific for girls. 


\section{Methods}

Sample

The TRacking Adolescents' Individual Lives Survey (TRAILS) is a prospective cohort study of Dutch (early) adolescents, with the aim to chart and explain the development of mental health from early adolescence into adulthood, both at the level of psychopathology and the levels of underlying vulnerability and environmental risk. Early adolescents will be measured biennially at least until they are 25 years. The present study involves data from the first (T1) assessment wave of TRAILS, which ran from March 2001 to July 2002. If both parents and early adolescents agreed to participate, parental written informed consent was obtained after the procedures had been fully explained. The study was approved by the National Dutch Medical Ethics Committee. Of all early adolescents approached for enrollment in the study $(N=3,145), 76.0 \%$ $(N=2,230$, mean age $=11.09, \mathrm{SD}=0.56,50.8 \%$ girls $)$ early adolescents participated in the study. Responders and non-responders did not differ with respect to the prevalence of teacher-rated behavior problems, nor regarding associations between sociodemographic variables and mental health outcomes. Detailed information about sample selection and analyses of non-response bias has been reported elsewhere [14, 23].

\section{Procedure}

Well-trained interviewers visited one of the parents or guardians (preferably the mother, 95.6\%) at their homes to administer an interview covering a wide range of topics, including developmental history and somatic health, parental psychopathology and care utilization. In addition to the interview, the parent was asked to fill out some questionnaires concerning the adolescent's mental health and behavior. Early adolescents filled out questionnaires at school, in the classroom, under the supervision of one or more TRAILS assistants. Besides, intelligence and a number of biological and neurocognitive parameters were assessed individually (at school, except for saliva samples, which were collected at home). Teachers were asked to fill out a brief questionnaire for all TRAILS participants in their class. Measures that were used in the present study are described more extensively below.

\section{Measures}

\section{Behavioral problems}

Behavioral problems were assessed with the Child Behavior Checklist (CBCL) $[2,48]$ and the Youth Self-
Report (YSR) [3, 49]. The CBCL is a measure of parentreported emotional and behavioral problems in 4- to 18year-old children and the YSR is a self-report questionnaire that was modeled on the CBCL. The CBCL and the YSR contain 113 and 112 items, respectively. These items are rated as 0 (not true), 1 (somewhat or sometimes true) or 2 (very true or often true). Both the CBCL and the YSR contain two broadband scales: one for internalizing behavior problems and one for externalizing behavior problems. In the present study, we used the mean of the standardized CBCL and YSR scores on externalizing behavior problems.

\section{Obstetric complications (OCs)}

Obstetric complications are defined as the broad class of deviations from the expected, normal course of events, including child development during pregnancy, labor/ delivery, and the early neonatal period [31]. Data concerning OCs were assessed in the parent interview. OCs included the presence of pregnancy complications (i.e. physical, social or psychological problems during pregnancy), complicated deliveries (i.e. breech presentation, Cesarean section), and hospitalization of the mother (i.e. due to physical problems, postnatal depression) or child (i.e. lack of oxygen, blood transfusion, jaundice) [17, 33]. A composite score on OCs was calculated on the basis of a list of 31 OCs (range $0-14$, mean $=1.87, \mathrm{SD}=2.19$ ). If no information was available for six or more items, cases were excluded from further analyses. The distribution of OCs is positively skewed, as most parents report $0(37.9 \%$, $N=609), 1(19.3 \%, N=309)$ or $2(15.4 \%, N=247)$ OCs.

\section{Cortisol}

TRAILS participants collected cortisol samples (saliva) at home, using the Salivette sampling device (Sarstedt, Rommelsdorfer Str., D-51588 Nümbrecht, Germany), which was handed to the parent at the parent interview, accompanied by a verbal and a written instruction. The Salivette tube consists of a plastic sampling vessel with a suspended insert containing a sterile neutral cotton wool swab that has to be chewed for about $45 \mathrm{~s}$ and then returned to the insert. Participants were instructed to collect three saliva samples: the first sample shortly after waking up (still lying in bed), the second sample 30 min later, and the third sample at 20:00 h. Both the sampling and the preceding day should be normal (school) days, without special events or stressful circumstances. Because the TRAILS participating schools started at approximately the same time, the sampling-time variation of the morning samples among the early adolescents is expected to be 
limited and the estimated corresponding times are $0700 \mathrm{~h}$ for the first sample $\left(\mathrm{Cort}_{0700}\right)$ and $0730 \mathrm{~h}$ for the second sample $\left(\mathrm{Cort}_{0730}\right)$. Participants were instructed not to collect saliva when they were ill, had a cold, had a headache, or were menstruating. Furthermore, they were requested not to take any medication, if possible. Any deviations from this protocol, either in terms of sampling times or in terms of other requirements, were indicated on an accompanying form. Concerning the sampling procedure itself, subjects were instructed to keep a glass of water next to their bed and to thoroughly rinse their mouth with tap water before sampling saliva, and not to consume sour products or brush their teeth shortly before that. Saliva samples were stored by the participants in their freezer directly after sampling and mailed to the institute as soon as possible. Participants who did not return the salivettes within a couple of months were sent a reminder letter. In total, we received saliva samples of 1,768 early adolescents (79.3\% of all TRAILS participants). Non-responders did not differ from responders in terms of gender (48.4 male vs. $49.4 \%$ male for non-responders vs. responders, respectively, $\chi^{2}$ $(d f=1)=0.132 ; P=0.716)$, OCs $(1.67$ vs. $1.73, t=$ $-0.569 ; P=0.569$ ), and externalizing behavior problems (0.51 vs. $0.51, t=-0.139 ; P=0.890$ ).

The saliva samples were stored at $-20^{\circ} \mathrm{C}$ until analysis. Previous studies suggest that salivary cortisol levels are stable for prolonged periods of time at $-20^{\circ} \mathrm{C}$ [1]. After completion of the data collection, all samples were sent in one batch (frozen, by courier) to the laboratory (Department of Clinical and Theoretical Psychobiology, University of Trier, Germany) for analysis. Procedures of determination of cortisol levels are described more extensively elsewhere [41].

\section{Statistical analyses}

A total of 82 early adolescents (4.6\%) used medication. Of these, we excluded 22 early adolescents because they used corticosteroid-containing medication. Based on the literature and based on their cortisol values, we found no reason to exclude any of the other participants who used medication. For each time point, single cortisol samples with values that were above $3 \mathrm{SD}$ of the mean of the particular time point were excluded from the analysis in order to reduce the impact of outliers (Cort ${ }_{0700} 21$ excluded, 1,666 valid measurements in the final dataset; Cort 073011 excluded, 1683 valid measurements in the final dataset; Cort $_{2000} 18$ excluded, 1,689 valid measurements in the final dataset). After this exclusion, cortisol levels followed a normal distribution (Cort ${ }_{0700}$ skewness, 0.700; kurtosis, 0.632; Cort $_{0730}$ skewness, 0.426, kurtosis, 0.239; Cort 2000 skewness, 1.217, kurtosis 2.014).
With regard to the morning cortisol levels, we used area under the curve (AUC) measures. The computation of the AUC is a frequently used method in endocrinological research to assess the overall secretion over a specific time period (AUC with respect to ground, $\mathrm{AUC}_{\mathrm{G}}$ ), and to estimate circadian changes over a specific time period (AUC with respect to increase, $\mathrm{AUC}_{\mathrm{I}}$ ) [39]. Pruessner et al. [39] recommend employing both formulas when analyzing datasets with repeated measures. We used the following formulas for calculating the (1) total cortisol levels after awakening (in $\mathrm{h} \times \mathrm{nmol} / \mathrm{l}$ ): $\mathrm{AUC}_{\mathrm{G}}=\left(\right.$ Cort $_{0730}-$ Cort $\left._{0700}\right) \quad 0.5 / 2+$ Cort $_{0700} \times 0.5$ (in which 0.5 refers to $0.5 \mathrm{~h}$ ) and (2) CAR (in $\mathrm{h} \times \mathrm{nmol} / \mathrm{l}$ ): $\mathrm{AUC}_{\mathrm{I}}=\left(\mathrm{Cort}_{0730}-\mathrm{Cort}_{0700}\right) \times 0.5 / 2$ (in which 0.5 refers to $0.5 \mathrm{~h}$ ). The former correlates 0.71 with Cort $_{0700}$ and 0.86 with Cort $_{0730}$ and the latter is in this design mathematically equal to one-quarter of the difference between awakening level and level $30 \mathrm{~min}$ later [41]. According to their conceptual meaning, we used $\mathrm{AUC}_{\mathrm{G}}$ levels following previous studies on basal cortisol samples and $\mathrm{AUC}_{\mathrm{I}}$ levels following previous studies on the CAR. We calculated the $\mathrm{AUC}_{\mathrm{G}}$ and $\mathrm{AUC}_{\mathrm{I}}$ for 1,615 participants, from which we received both Cort $_{0700}$ and Cort 0730 . Furthermore, we used Cort $_{2000}$ levels for our interest in basal cortisol levels in the evening [41]. For 1,689 participants, we received Cort $_{2000}$ levels.

In a previous study on the present sample, gender and the quadratic effect of sampling month were identified as significant predictors of HPA-axis activity. Age, pubertal development, and BMI appeared not to be related to HPA-axis activity [41]. Prior to analyses, all predictor variables were standardized to minimize multicollinearity. Following the framework described by Baron and Kenny [6], we tested for potential mediation of HPA-axis activity by a series of four regression analyses. In the first three regression analyses, each of the HPA-axis measures (i.e. $\mathrm{AUC}_{\mathrm{G}}, \mathrm{AUC}_{\mathrm{I}}$ and $\mathrm{Cort}_{2000}$ ) were the dependent variable. In these regression analyses, the quadratic effect of sampling month was entered in the first block of the model. OCs, gender, and the interaction term between OCs and gender, were entered in the second block of the model. In the fourth regression analysis, externalizing behavior was the dependent variable. Owing to its potential confounding effect, socioeconomic status was entered in the first block of the model. Again, OCs, gender, and the interaction term between OCs and gender, were entered in the second block of the model. If OCs were a significant predictor of both HPA-axis measure and externalizing behavior problems, externalizing behavior problems were regressed on both HPA-axis measure and OCs in a fifth regression. If any of the 
Table 1 Mean and standard deviation of the (unstandardized) variables for the total group and by gender

\begin{tabular}{|c|c|c|c|c|c|c|c|c|c|c|c|}
\hline & \multicolumn{3}{|l|}{ Total } & \multicolumn{3}{|l|}{ Boys } & \multicolumn{3}{|l|}{ Girls } & \multicolumn{2}{|c|}{ Boys versus Girls } \\
\hline & $n$ & Mean & (SD) & $n$ & Mean & (SD) & $n$ & Mean & (SD) & $t$ & $P$ \\
\hline Externalizing behavior & 2,000 & 0.51 & $(0.32)$ & 980 & 0.58 & $(0.35)$ & 1,020 & 0.44 & $(0.28)$ & -9.8 & $<0.001$ \\
\hline OCs & 2,166 & 1.72 & $(2.01)$ & 1,061 & 1.80 & $(2.09)$ & 1,105 & 1.63 & $(1.93)$ & -2.0 & 0.05 \\
\hline \multicolumn{12}{|l|}{ HPA-axis activity } \\
\hline $\mathrm{AUC}_{\mathrm{G}}$ & 1,615 & 6.72 & $(2.24)$ & 803 & 6.48 & $(2.21)$ & 812 & 6.95 & $(2.24)$ & 4.3 & $<0.001$ \\
\hline $\mathrm{AUC}_{\mathrm{I}}$ & 1,615 & 0.95 & $(1.73)$ & 803 & 0.88 & (1.64) & 812 & 1.01 & $(1.82)$ & 1.5 & 0.13 \\
\hline Cort $_{2000}$ & 1,689 & 1.95 & $(1.33)$ & 835 & 1.90 & (1.33) & 854 & 2.00 & $(1.34)$ & 1.5 & 0.14 \\
\hline
\end{tabular}

Externalizing behavior mean scores on CBCL and YSR (range 0-2), OCs total obstetric complications

$\mathrm{AUC}_{\mathrm{G}}$ and $\mathrm{AUC}_{\mathrm{I}}$ in $\mathrm{h} \times \mathrm{nmol} / \mathrm{l}$ and $\mathrm{Cort}_{2000}$ in nmol/l. The last column lists the results of the independent samples $t$ tests comparing boys and girls

interaction terms with gender were significant, we performed gender-stratified analyses.

\section{Results}

Table 1 shows the mean and standard deviation of the (unstandardized) variables for the total group and by gender. As expected, boys showed more externalizing behavior problems and OCs than girls. In addition, girls showed higher $\mathrm{AUC}_{\mathrm{G}}$ levels than boys, while $\mathrm{AUC}_{\mathrm{I}}$ levels and Cort $_{2000}$ levels did not differ between boys and girls.

\section{Testing mediation}

Table 2 shows the results of the regression analyses testing our mediation hypothesis. OCs were not a significant predictor of $\mathrm{AUC}_{\mathrm{G}}$ levels, $\mathrm{AUC}_{\mathrm{I}}$ levels or Cort $_{2000}$ levels. As in previous TRAILS studies [30, 41], gender predicted $\mathrm{AUC}_{\mathrm{G}}$ levels $(\beta=-0.098, P<0.001)$ and the quadratic effect of sampling month predicted AUC $_{\mathrm{G}}$ levels $(\beta=0.112, \quad P<0.001), \quad \mathrm{AUC}_{\mathrm{I}}$ levels $(\beta=0.072, P<0.01)$, and Cort $_{2000}$ levels $(\beta=0.203$, $P<0.001)$.

Socioeconomic status (SES) significantly predicted externalizing behavior problems $(\beta=-0.150, P<0.001)$ and accounted for $2.4 \%$ of the adjusted variance. In addition, OCs $(\beta=0.052, P<0.05)$ and gender $(\beta=0.210$, $P<0.001)$ predicted externalizing behavior problems. The effects of SES, OCs and gender accounted for $7.0 \%$ of the adjusted variance.

Because none of the interaction terms with gender were significant, we did not perform gender-stratified analyses. Given the requirement that the independent variable (OCs) must affect the mediator (HPA-axis activity) to establish mediation [6], HPA-axis activity did not emerge as a
Table 2 Regression analyses testing for mediation

\begin{tabular}{lllll}
\hline Predictor & Dependent & Beta & $P$ value & Adj. $R^{2}(\%)$ \\
\hline Model 1 & AUC $_{\mathrm{G}}$ & & & \\
Block 1 & & & & \\
Sampling month & & 0.112 & $<0.001$ & 1.2 \\
Block 2 & & & \\
OCs & & -0.029 & 0.25 & \\
Gender & -0.098 & $<0.001$ & 2.1 \\
OCs $\times$ gender & & 0.019 & 0.44 &
\end{tabular}

Model $2 \quad$ AUC $_{\mathrm{I}}$

Block 1

Sampling month

$0.072<0.01 \quad 0.5$

Block 2

OCs

$-0.007 \quad 0.78$

Gender

$-0.035 \quad 0.17$

OCs $\times$ gender

$-0.024 \quad 0.33$

Model 3

Block 1

$0.203<0.001 \quad 4.0$

Sampling month

Block 2

$\begin{array}{lll}\text { OCs } & -0.040 & 0.10 \\ \text { Gender } & -0.026 & 0.29 \\ \text { OCs } \times \text { gender } & -0.030 & 0.22\end{array}$

Model 4

Externalizing

Block 1

SES

$-0.150<0.001 \quad 2.4$

Block 2

OCs

$\begin{array}{lll}0.052 & 0.02 \quad 7.0\end{array}$

Gender

$0.210<0.001 \quad 7.0$

OCs $\times$ gender

$0.017 \quad 0.81$

Sampling month quadratic effect of sampling month, OCs obstetric complications, SES socioeconomic status, Externalizing externalizing behavior problems. OCs, gender, $\mathrm{AUC}_{\mathrm{G}}, \mathrm{AUC}_{\mathrm{I}}$, Cort2000, and SES are standardized variables. Adjusted $R^{2}$ is reported for significant effects 
mediator of the relation between OCs and externalizing behavior problems.

\section{Discussion}

There are empirical and theoretical reasons to expect that HPA-axis activity serves as a mediator in the relationship between OCs and externalizing behavior problems. The present study is the first that investigates this mediation model in a large population-based sample of early adolescents. We found that HPA-axis did not mediate the relationship between OCs and externalizing behavior problems.

Although we did not find a mediating effect of HPA-axis activity, our mediator analyses did reveal a direct relationship between OCs and externalizing behavior problems. Consistent with many previous studies $[5,7,21$, 35, 40], including a TRAILS study [10], a history of OCs was identified as an environmental risk factor for externalizing behavior problems. This effect was independent of the effect of gender, suggesting that the effect of OCs was not due to the fact that boys experienced more OCs than girls. It must be noted, however, that the effect size of the predictive effect of OCs on externalizing behavior problems is small. Together with gender, which clearly accounted for most of the adjusted variance, OCs predicted only $4.6 \%$ of the adjusted variance. In addition, the relationship between OCs and externalizing behavior problems was not gender-specific. This is in line with a study by Batstra et al. [7] who found that suboptimal obstetric conditions were related to externalizing behavior problems in both boys and girls. In contrast, Allen et al. [5] suggested that the association between OCs and externalizing behavior problems may be specific for girls. However, their finding may be the result of a type I error resulting from multiple testing [5].

We adopted a model inspired by Allen et al. [5] who suggested a biological model in which neurobiological deficits mediate the relationship between OCs and externalizing behavior problems. They found that this model was particularly appropriate for explaining the relationship between OCs and externalizing behavior problems, as other models were more appropriate for explaining the relationship between OCs and internalizing behavior problems. In addition, this model is very similar to (a part of) the model described by Raine [40] and van Goozen et al. [47]. Both studies focused on explaining the relationship between early risk factors and antisocial behavior. Why is it then that we did not find a mediating effect? Although we found a gender-specific relationship between HPA-axis activity and externalizing behavior problems in our previous study [30], there was no evidence of a relationship between OCs and HPA-axis activity. The latter is in contrast to previous studies showing that early adversities may adversely affect the function of the fetal and neonatal HPA-axis [11, 24, 32, $36,44]$, leading to elevated cortisol levels [11, 24, 32, 36]. Yet, previous studies have reported on specific OCs, while no study, to our knowledge, has reported on composite scores on OCs in relation to HPA-axis activity. We were unable to test the possibility that specific OCs were related to HPA-axis activity as the number of early adolescents that had a specific OC item was often too small to draw conclusions about that single item. It is also possible that OCs are related to HPA-axis activity, but not to the cortisol measures in our sample. First, concerning the increase in cortisol levels after awakening (CAR or AUC $\mathrm{I}_{\mathrm{I}}$ levels), only one study found an indication that maternal anxiety during pregnancy may lead to an elevated CAR in pre-adolescent children [36]. However, the significance of findings with regard to the CAR is difficult to determine since studies often use different methodologies [12]. Thus, more research is needed to assess the relationship between OCs and the CAR, using similar methodology to determine the CAR. Secondly, we were also unable to find a relationship between OCs and evening cortisol levels. O'Connor et al. [36] did not find an association between maternal anxiety during pregnancy and evening cortisol levels either. Reason for this non-finding may be that evening cortisol levels display little inter-subject variability [41], possibly making it more difficult to detect associations. Finally, we did not find a relationship between OCs and total cortisol levels after awakening ( $\mathrm{AUC}_{\mathrm{G}}$ levels) despite other studies that found an association between specific OCs and elevated cortisol levels measured in pre-adolescence [11, 36]. The fact that these studies found elevated cortisol levels in preadolescence $[11,36]$ seems to exclude the possibility that OCs initially provoke elevations in cortisol, which after downregulation of the HPA-axis decrease to lower cortisol levels as a long term consequence $[13,18,20]$. Still, specific OCs may provide the most valid explanation for not finding a relationship between OCs and HPA-axis activity. Future research on high-risk samples may provide useful information on this possibility. If OCs do not account for differences in HPA-axis activity, there may be other risk factors such as life events and family and parenting factors that affect HPA-axis activity.

It is still possible that brain damage is a mediator in the relationship between OCs and externalizing behavior problems [5], as HPA-axis is not the only possible mediator in this relationship. One possibility is that OCs lead to neurological impairment in the frontal lobes, which are essential for executive functioning [9]. In turn, executive function deficits may predispose to externalizing behavior problems [40]. A second possibility is that OCs lead to other forms of brain damage, which indirectly leads to 
externalizing behavior problems. For example, when brain damage causes a physical disability, a child may become less confident in interaction with other children, which then in turn elevates the risk for externalizing behavior problems [5]. A third possibility is that neurobiological deficits may interact with other factors such as cognitive and emotional functioning [47] and social risk factors [40]. In addition, androgens and the autonomic nervous system may function as potential mediators [47]. All in all, we can conclude that the potential biological mediation of the relationship between OCs and externalizing behavior problems is far more complex than we assumed.

A major strength of the present study is that it was based on a very large population-based sample of early adolescents. This enabled us to test the mediating effect of HPA-axis activity on the relationship between OCs and externalizing behavior problems without the influence of selection biases which are inherent in clinically referred samples [14]. A potential limitation concerns the retrospective nature of the information on OCs. However, a previous study concluded that maternal recall may be a surprisingly accurate source of obstetric information and hence an acceptable alternative to more objective indices in a prospective study [5]. Another limitation concerns the cortisol sampling. First, home collection of saliva is much more susceptible to situational influences than collection of saliva in the more controlled conditions at the laboratory. In addition, home collection relies heavily upon participant adherence [12]. Recent research suggests, however, that home assessment of cortisol in saliva provides the same results as the assessment under highly controlled laboratory conditions [50]. Secondly, the present study involves only one day of cortisol assessment. However, we are still confident in the reliability of our cortisol data. One reason is that the CAR (both overall activity and dynamic of the response) is reasonably stable for individuals across days $[12,51]$. In addition, both the sampling and the preceding day were normal school days. In a sample of adults, Hellhammer et al. [22] revealed that sampling on workdays may reduce the within-subject variation of situational factors because of the uniform schedule on workdays. Since school days are highly scheduled, it is probable that the same applies to the early adolescents in our sample. Moreover, the possible reduction in reliability as a result of one day of cortisol assessment may be counterbalanced by the sample size, which is large enough to off set random fluctuations in individual values.

\section{Conclusions}

In addition to our previous study which demonstrated a relationship between HPA-axis activity and externalizing behavior problems in girls [30], we confirmed the relationship between OCs and externalizing behavior problems [10], although the effect size of this relationship is small. However, these two mechanisms were not related to each other, indicating that HPA-axis activity was not a mediator in the relationship between OCs and externalizing behavior problems. Future research should focus on understanding the mechanism through which OCs cause externalizing behavior problems.

Acknowledgments This research is part of the Tracking Adolescents' Individual Lives Survey (TRAILS). Participating centers of TRAILS include various departments of the University Medical Center Groningen and University of Groningen, the Erasmus University Medical Center Rotterdam, the University of Utrecht, the Radboud Medical Center Nijmegen, and the Trimbos Institute, all in the Netherlands. Principal investigaters are prof. Dr. J. Ormel (University Medical Center Groningen) and prof. Dr. F.C. Verhulst (Erasmus University Medical Center Rotterdam). TRAILS has been financially supported by various grants from the Netherlands Organization for Scientific Research NOW (Medical Research Council program grant GB-MW 940-38-011; ZonMW Brainpower grant 100001-004; ZonMW Risk Behavior and Dependence grant 60-6060097-118; ZonMW Culture and Health grant 261-98-710; Social Sciences Council medium-sized investment grant GB-MaGW 48001-006 and project grants GB-MaGW 457-03-018 and GB-MaGW 452-04-314; NOW large-sized investment grant 175.010.2003.005); the Sophia Foundation for Medical Research (projects 301 and 393), the Dutch Ministry of Justice (WODC), and the participating universities. The funding sources had no further role in study design, analysis and interpretation of the data, and in writing of the report. We are grateful to all adolescents, their parents and teachers who participated in this research and to everyone who worked on this project and made it possible.

Open Access This article is distributed under the terms of the Creative Commons Attribution Noncommercial License which permits any noncommercial use, distribution, and reproduction in any medium, provided the original author(s) and source are credited.

\section{References}

1. Aardal E, Holm AC (1995) Cortisol in saliva-reference ranges and relation to cortisol in serum. Eur J Clin Chem Clin Biochem 33:927-932

2. Achenbach TM (1991) Manual for the Child Behavior Checklist/ 4-18 and 1991 Profile. Department of Psychiatry, University of Vermont, Burlington, Vermont

3. Achenbach TM (1991) Manual for the Youth Self-Report and 1991 Profile. Department of Psychiatry, University of Vermont, Burlington, Vermont

4. Alink LR, van IJzendoorn MH, Bakermans-Kranenburg MJ, Mesman J, Juffer F, Koot HM (2008) Cortisol and externalizing behavior in children and adolescents: mixed meta-analytic evidence for the inverse relation of basal cortisol and cortisol reactivity with externalizing behavior. Dev Psychobiol 50:427450

5. Allen NB, Lewinsohn PM, Seeley JR (1998) Prenatal and perinatal influences on risk for psychopathology in childhood and adolescence. Dev Psychopathol 10:513-529 
6. Baron RM, Kenny DA (1986) The moderator-mediator variable distinction in social psychological research: conceptual, strategic, and statistical considerations. J Pers Soc Psychol 51:1173-1182

7. Batstra L, Hadders-Algra M, Ormel J, Neeleman J (2004) Obstetric optimality and emotional problems and substance use in young adulthood. Early Hum Dev 80:91-101

8. Batstra L, Neeleman J, Elsinga C, Hadders-Algra M (2006) Psychiatric morbidity is related to a chain of prenatal and perinatal adversities. Early Hum Dev 82:721-729

9. Beck JE, Shaw DS (2005) The influence of perinatal complications and environmental adversity on boys' antisocial behavior. J Child Psychol Psychiatry 46:35-46

10. Buschgens CJ, Swinkels SH, van Aken MA, Ormel J, Verhulst FC, Buitelaar JK (2009) Externalizing behaviors in preadolescents: familial risk to externalizing behaviors, prenatal and perinatal risks, and their interactions. Eur Child Adolesc Psychiatry 18:65-74

11. Buske-Kirschbaum A, Krieger S, Wilkes C, Rauh W, Weiss S, Hellhammer DH (2007) Hypothalamic-pituitary-adrenal axis function and the cellular immune response in former preterm children. J Clin Endocrinol Metab 92:3429-3435

12. Clow A, Thorn L, Evans P, Hucklebridge F (2004) The awakening cortisol response: methodological issues and significance. Stress 7:29-37

13. De Bellis MD, Baum AS, Birmaher B, Keshavan MS, Eccard CH, Boring AM, Jenkins FJ, Ryan ND (1999) A.E. Bennett Research Award. Developmental traumatology. Part I: Biological stress systems. Biol Psychiatry 45:1259-1270

14. de Winter AF, Oldehinkel AJ, Veenstra R, Brunnekreef JA, Verhulst FC, Ormel J (2005) Evaluation of non-response bias in mental health determinants and outcomes in a large sample of pre-adolescents. Eur J Epidemiol 20:173-181

15. Deater-Deckard K, Plomin R (1999) An adoption study of the etiology of teacher and parent reports of externalizing behavior problems in middle childhood. Child Dev 70:144-154

16. Entringer S, Kumsta R, Hellhammer DH, Wadhwa PD, Wüst S (2008) Prenatal exposure to maternal psychosocial stress and HPA axis regulation in young adults. Horm Behav 55(2):292298

17. Gillberg C (1995) Clinical child neuropsychiatry. Cambridge University Press, Cambridge

18. Gunnar MR, Donzella B (2002) Social regulation of the cortisol levels in early human development. Psychoneuroendocrinology 27:199-220

19. Gunnar MR, Fisher PA (2006) Bringing basic research on early experience and stress neurobiology to bear on preventive interventions for neglected and maltreated children. Dev Psychopathol 18:651-677

20. Gunnar MR, Vazquez DM (2001) Low cortisol and a flattening of expected daytime rhythm: potential indices of risk in human development. Dev Psychopathol 13:515-538

21. Gutteling BM, de Weerth C, Willemsen-Swinkels SH, Huizink AC, Mulder EJ, Visser GH, Buitelaar JK (2005) The effects of prenatal stress on temperament and problem behavior of 27month-old toddlers. Eur Child Adolesc Psychiatry 14:41-51

22. Hellhammer J, Fries E, Schweisthal OW, Schlotz W, Stone AA, Hagemann D (2007) Several daily measurements are necessary to reliably assess the cortisol rise after awakening: state- and trait components. Psychoneuroendocrinology 32:80-86

23. Huisman M, Oldehinkel AJ, de Winter A, Minderaa RB, de Bildt A, Huizink AC, Verhulst FC, Ormel J (2008) Cohort profile: the Dutch 'TRacking Adolescents' Individual Lives' Survey'; TRAILS. Int J Epidemiol 37:1227-1235

24. Huizink AC, Mulder EJ, Buitelaar JK (2004) Prenatal stress and risk for psychopathology: specific effects or induction of general susceptibility? Psychol Bull 130:115-142
25. Huizink AC, Robles de Medina PG, Mulder EJ, Visser GH, Buitelaar JK (2003) Stress during pregnancy is associated with developmental outcome in infancy. J Child Psychol Psychiatry 44:810-818

26. Jester JM, Nigg JT, Adams K, Fitzgerald HE, Puttler LI, Wong MM, Zucker RA (2005) Inattention/hyperactivity and aggression from early childhood to adolescence: heterogeneity of trajectories and differential influence of family environment characteristics. Dev Psychopathol 17:99-125

27. Klimes-Dougan B, Hastings PD, Granger DA, Usher BA, ZahnWaxler C (2001) Adrenocortical activity in at-risk and normally developing adolescents: individual differences in salivary cortisol basal levels, diurnal variation, and responses to social challenges. Dev Psychopathol 13:695-719

28. Kraemer S (2000) The fragile male. Br Med J 321:1609-1612

29. Liu J (2004) Childhood externalizing behavior: theory and implications. J Child Adolesc Psychiatr Nurs 17:93-103

30. Marsman R, Swinkels SH, Rosmalen JG, Oldehinkel AJ, Ormel J, Buitelaar JK (2008) HPA-axis activity and externalizing behavior problems in early adolescents from the general population: the role of comorbidity and gender. The TRAILS study. Psychoneuroendocrinology 33:789-798

31. McNeil TF (1988) Obstetric factors and perinatal injuries. In: Tsuang MT, Simpson JC (eds) Handbook of schizophrenia, nosology, epidemiology and genetics, vol 3. Elsevier, Amsterdam, pp 319-344

32. Mears K, McAuliffe F, Grimes H, Morrison JJ (2004) Fetal cortisol in relation to labour, intrapartum events and mode of delivery. J Obstet Gynaecol 24:129-132

33. Milberger S, Biederman J, Faraone SV, Guite J, Tsuang MT (1997) Pregnancy, delivery and infancy complications and attention deficit hyperactivity disorder: issues of gene-environment interaction. Biol Psychiatry 41:65-75

34. Moffitt TE, Caspi A (2001) Childhood predictors differentiate life-course persistent and adolescence-limited antisocial pathways among males and females. Dev Psychopathol 13:355-375

35. Nosarti C, Allin MP, Frangou S, Rifkin L, Murray RM (2005) Hyperactivity in adolescents born very preterm is associated with decreased caudate volume. Biol Psychiatry 57:661-666

36. O'Connor TG, Ben-Shlomo Y, Heron J, Golding J, Adams D, Glover V (2005) Prenatal anxiety predicts individual differences in cortisol in pre-adolescent children. Biol Psychiatry 58:211-217

37. Pajer K, Gardner W, Rubin RT, Perel J, Neal S (2001) Decreased cortisol levels in adolescent girls with conduct disorder. Arch Gen Psychiatry 58:297-302

38. Prechtl HF (1980) The optimality concept. Early Hum Dev 4:201-205

39. Pruessner JC, Kirschbaum C, Meinlschmid G, Hellhammer DH (2003) Two formulas for computation of the area under the curve represent measures of total hormone concentration versus timedependent change. Psychoneuroendocrinology 28:916-931

40. Raine A (2002) Biosocial studies of antisocial and violent behavior in children and adults: a review. J Abnorm Child Psychol 30:311-326

41. Rosmalen JG, Oldehinkel AJ, Ormel J, de Winter AF, Buitelaar JK, Verhulst FC (2005) Determinants of salivary cortisol levels in 10-12 year old children: a population-based study of individual differences. Psychoneuroendocrinology 30:483-495

42. Sandberg S (2002) Hyperactivity and attention disorders of childhood. Cambridge University Press, Cambridge

43. Sondeijker FE, Ferdinand RF, Oldehinkel AJ, Veenstra R, Tiemeier H, Ormel J, Verhulst FC (2007) Disruptive behaviors and HPA-axis activity in young adolescent boys and girls from the general population. J Psychiatr Res 41:570-578

44. Taylor A, Fisk NM, Glover V (2000) Mode of delivery and subsequent stress response. Lancet 355:120 
45. Tsigos C, Chrousos GP (2002) Hypothalamic-pituitary-adrenal axis, neuroendocrine factors and stress. J Psychosom Res 53:865871

46. van Bokhoven I, van Goozen SH, van Engeland H, Schaal B, Arseneault L, Seguin JR, Nagin DS, Vitaro F, Tremblay RE (2005) Salivary cortisol and aggression in a population-based longitudinal study of adolescent males. J Neural Transm 112:1083-1096

47. van Goozen SH, Fairchild G, Snoek H, Harold GT (2007) The evidence for a neurobiological model of childhood antisocial behavior. Psychol Bull 133:149-182

48. Verhulst FC, Van der Ende J, Koot HM (1996) Handleiding voor de CBCL/4-18. Afdeling Kinder- en jeugdpsychiatrie, Sophia
Kinderziekenhuis/Academisch Ziekenhuis Rotterdam/Erasmus Universiteit Rotterdam, Rotterdam

49. Verhulst FC, Van der Ende J, Koot HM (1997) Handleiding voor de Youth Self-Report (YSR). Afdeling Kinder- en jeugdpsychiatrie, Sophia Kinderziekenhuis/Academisch Ziekenhuis Rotterdam/Erasmus Universiteit Rotterdam, Rotterdam

50. Wilhelm I, Born J, Kudielka BM, Schlotz W, Wüst S (2007) Is the cortisol awakening rise a response to awakening? Psychoneuroendocrinology 32:358-366

51. Wüst S, Wolf J, Hellhammer DH, Federenko I, Schommer N, Kirschbaum C (2000) The cortisol awakening response-normal values and confounds. Noise Health 2:79-88 